\title{
Fabrication of functionalized halloysite nanotube blended ultrafiltration membranes for high flux and fouling resistance
}

\author{
Shinyun Park ${ }^{1,2}$, Eunmok Yang ${ }^{1,2}$, Hosik Park $^{2^{\dagger}}$, Heechul Choi ${ }^{{ }^{\dagger}}$ \\ ${ }^{1}$ School of Environmental Science and Engineering, Gwangju Institute of Science and Technology (GIST), Gwangju 61005, Republic of Korea \\ ${ }^{2}$ Center for membranes, Advanced Materials Division, Korea Research Institute of Chemical Technology (KRICT), Daejeon, 44412, Republic of Korea
}

\begin{abstract}
Halloysite nanotubes (HNTs) were functionalized using 3-aminopropyltriethoxysilane (APTES) and incorporated into polyethersulfone (PES) membranes to improve the hydrophilicity of the membranes as well as the interfacial interaction between HNTs and the polymer matrix. The intrinsic properties, permeability, and selectivity of the prepared membranes were analyzed to evaluate the membrane performance. In addition, humic acid (HA) fouling experiments were conducted to measure the antifouling properties of the fabricated membranes. As HNTs and functionalized HNTs (f-HNTs) contents are increased, hydrophilicity and mechanical strength were enhanced, and membranes with f-HNTs showed further improved performance. The pure water flux of membranes with $2 \%$ HNTs and f-HNT was 7.5 times higher than that of a pristine PES membrane without a trade-off relation between the water flux and HA rejection. The membranes with f-HNTs showed the highest antifouling properties compared to membranes with HNTs because enhanced hydrophilicity played a key role in preventing accumulation of HA.
\end{abstract}

Keywords: Antifouling, Halloysite nanotube (HNT), Membrane, Ultrafiltration, 3-aminopropyltriethoxysilane (APTES)

\section{Introduction}

The global population has steadily increased in tandem with industrialization and urbanization. According to the world population clock, the population is projected rise from the current level of 7.6 billion to reach 10 billion by 2050 [1, 2]. The demand for potable water is rising, due to population growth, contamination of water resources, and climate change. Thus, there is a need for an approach to purify water at lower cost, using less energy and minimizing the impact on the environment [3].

Membrane technologies have been demonstrated by many researchers as a cost effective method with high water quality and excellent separation efficiency [3]. However, some disadvantages still remain such as membrane fouling, low water production, and short life time. Methods to overcome these problems such as grafting, surface coating and blending additives have been developed. One way to minimize surface fouling and increase the water production is to incorporate nanomaterials into the membranes. Modifying the membranes by blending organic and inorganic nanomaterials may offer advantages such as increased filtration performance, improved mechanical properties, and better

This is an Open Access article distributed under the terms of the Creative Commons Attribution Non-Commercial License (http://creativecommons.org/licenses/by-nc/3.0/) which permits unrestricted non-commercial use, distribution, and reproduction in any medium, provided the original work is properly cited.

Copyright (C) 2020 Korean Society of Environmental Engineers antifouling capability [3, 4]. In particular, inorganic nanomaterials have received considerable attention owing to their physicochemical properties such as hydrophilicity, large surface area, and thermal and mechanical stability. Different nanomaterials have been used for membrane fabrication: carbon nanotubes (CNTs), graphene oxide (GO), aluminum oxide $\left(\mathrm{Al}_{2} \mathrm{O}_{3}\right)$, titanium oxide $\left(\mathrm{TiO}_{2}\right)$, silver (Ag), etc. [5-11]. Among these, halloysite nanotubes (HNTs) have been reported in the literature as promising nanofiller candidates due to their low production cost, less environmental risks, and unique structure [12].

HNTs, first named by Berthier in 1826, are chemically similar to Kaolinite since they are derived by the delamination and rolling of Kaolinite. HNTs possess a hollow tubular structure with multilayer aluminosilicates, and the gaps between the aluminosilicate layers are 7-10 $\AA$. The length of the HNTs is in a range of 0.5-10 $\mu \mathrm{m}$, and the inner and outer diameters are 30-50 nm and 50-100 $\mathrm{nm}$, respectively. The chemical formula of HNTs is $\mathrm{Al}_{2} \mathrm{Si}_{2} \mathrm{O}_{5}(\mathrm{OH})_{4}$. $\mathrm{nH}_{2} \mathrm{O}$, and the surface is composed of $\mathrm{Si}-\mathrm{OH}, \mathrm{Al}-\mathrm{OH}$ and $\mathrm{Si}-\mathrm{O}-\mathrm{Si}$ groups, which are hydrophilic and enable functionalization and modification [13, 14]. HNTs have excellent mechanical and physical properties, and halloysite nanotubes are thus promising nano-

Received October 4, 2019 Accepted October 17, 2019

${ }^{\dagger}$ Corresponding author

Email: hcchoi@gist.ac.kr (H.Choi), hspark@krict.re.kr (H.Park)

Tel: 82-62-715-2441 Fax: 82-62-715-2423

ORCID: 0000-0002-8132-0983 (H.Choi), 0000-0002-5151-1113 (H.Park) 
filler for membrane modification [15].

However, there are drawbacks that constrain the application of inorganic materials including HNTs with polymers, such as poor dispersibility and weak interfacial interaction between polymers and HNTs. [16]. In an attempt to address those problems, silane treatment has been applied on the surface of inorganic materials using a silane coupling agent, because it was reported that the functionalization of inorganic materials with hydrophilic groups such as amine, carboxylic acid, and sulfonic acid leads to membranes with higher surface hydrophilicity and increased mechanical strength [17]. Among various types of silane coupling agents, 3-aminopropyltriethoxysilane (APTES), which is composed of one ethoxy- group and three amino- groups with $\mathrm{Si}$, is the most extensively reported coupling agent in the literature based on its ability to provide hydrophilicity [14, 17].

In this study, APTES was used for the functionalization of HNTs and the functionalized HNTs were incorporated into a polyethersulfone (PES) matrix. APTES was used to improve the hydrophilicity and mechanical properties of the membrane. The membranes were prepared via the phase inversion method using solutions with different concentrations of HNTs and functionalized HNTs. The performance of the fabricated membranes including permeability, selectivity, and antifouling properties was evaluated and compared.

\section{Experimental}

\subsection{Functionalization of HNTs}

For the functionalization of HNTs, APTES (99\%; Sigma-Aldrich, USA) solution $(0.6 \%)$ was mixed with ethanol/water in a ratio of $6: 4$. For the hydrolysis of silane, the $\mathrm{pH}$ of the solution was adjusted between 3.5 and 4.5 by adding acetic acid (Glacial, 99.7\%; Daejung, Korea). The HNTs (Sigma-Aldrich, USA) were added to the solution and mixed using a stirrer for $2 \mathrm{~h}$ to obtain a homogeneous dispersion. The treated HNTs were dried in an oven at $100{ }^{\circ} \mathrm{C}$ until ethanol was completely removed, and finally functionalized HNTs (f-HNTs) were obtained. The interaction mechanism is shown in Fig. 1.

\subsection{Membrane Fabrication}

For membrane synthesis, HNTs and f-HNTs were added in n-methyl-2-pyrrolidinone (NMP) (anhydrous 99.5\%; Sigma-Aldrich, USA). And then, 1 wt.\% of polyvinylpyrrolidone (PVP) (Mw 10,000

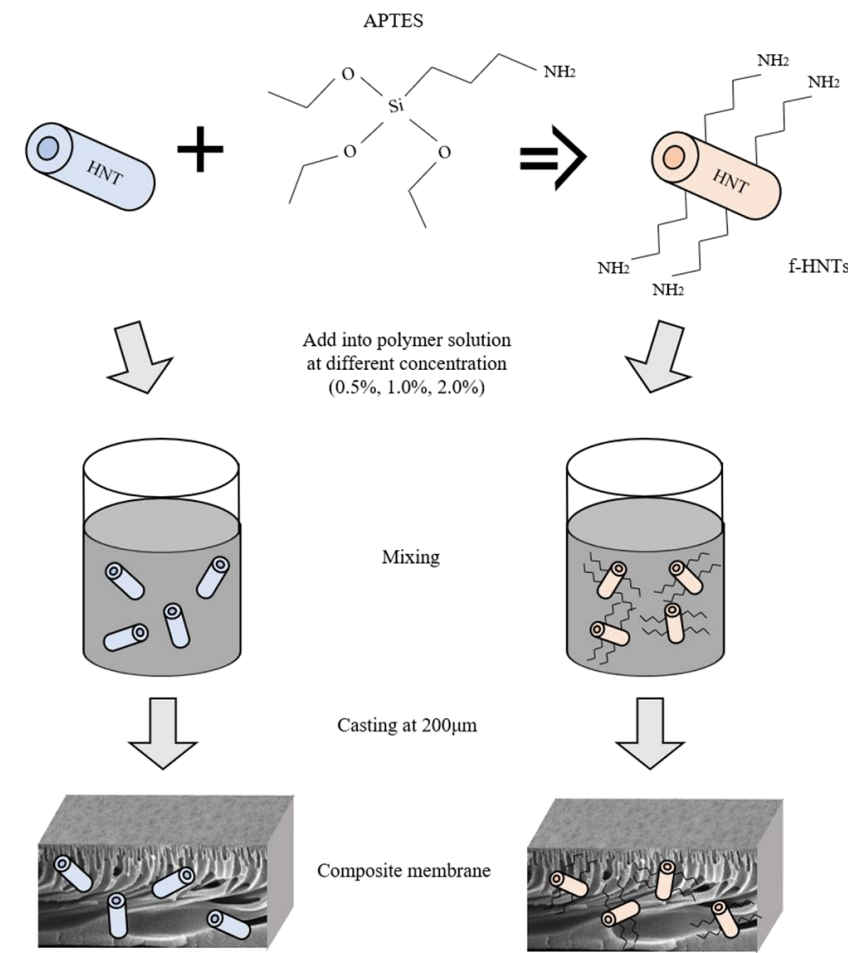

Fig. 1. Preparation process of ultrafiltration membranes with HNTs and f-HNTs.

g/mol; Sigma-Aldrich, USA), a hydrophilic pore-former, was added into NMP with HNTs and f-HNTs. Finally, 20 wt.\% of PES (Gafone 3000P; Solvay co., Korea), which was dried in an oven at $40{ }^{\circ} \mathrm{C}$ to remove all the moisture, was dissolved in the solution. The whole preparation step for the casting solution took $7 \mathrm{~d}$. The ratio of HNTs and f-HNTs was varied from 0.5 wt.\% to 2.0 wt.\% of PES by weight, as summarized in Table 1 . The prepared solution was sonicated for $3 \mathrm{~h}$ and degassed for $30 \mathrm{~min}$ to remove visible and invisible air bubbles.

The casting was carried out using a film applicator (Multi Applicator; TQC Sheen, Korea) at $200 \mu \mathrm{m}$ thickness on a glass plate under room ambient conditions. The casted membrane was immersed into a water bath after exposure to air for $30 \mathrm{~s}$. The synthesized membrane was separated from the glass plate, and then stored in deionized (DI) water till further use. DI water was obtained from a water purification system (Dream Plus II; MDM

Table 1. Detailed Composition of the Casting Solution for Membrane Synthesis

\begin{tabular}{|c|c|c|c|c|c|}
\hline Type of membrane & Polymer & Solvent & Additives & HNTs & f-HNTs \\
\hline PES & \multirow{7}{*}{$20 \%$} & \multirow{7}{*}{$79 \%$} & \multirow{7}{*}{$1 \%$} & - & - \\
\hline HNT0.5 & & & & $0.5 \%$ & - \\
\hline HNT1.0 & & & & $1.0 \%$ & - \\
\hline HNT2.0 & & & & $2.0 \%$ & - \\
\hline f-HNT0.5 & & & & - & $0.5 \%$ \\
\hline f-HNT1.0 & & & & - & $1.0 \%$ \\
\hline f-HNT2.0 & & & & - & $2.0 \%$ \\
\hline
\end{tabular}


co., Korea) with a resistivity of $18.2 \mathrm{~m} \Omega-\mathrm{cm}$. The schematic membrane fabrication process is shown in Fig. 1.

\subsection{Characterizations}

Fourier transform infrared (FTIR) spectroscopy (Spectrum 400; PerkinElmer, Korea) was used to obtain the surface chemistry of HNTs and f-HNTs. The spectra were recorded at wavelength from $4,000 \mathrm{~cm}^{-1}$ to $450 \mathrm{~cm}^{-1}$. X-ray photoelectron spectroscopy (XPS) (K-Alpha ${ }^{+}$; Thermo fisher, USA) was used to analyze the elemental composition of the surface of HNTs and f-HNTs. The spectra were recorded with binding energies from $600 \mathrm{eV}$ to $0 \mathrm{eV}$.

The morphology of the HNTs, f-HNTs, and the fabricated membranes were observed with the use of field-emission scanning electron microscopy (FE-SEM) (JSM-7500F; JEOL, Korea) at different magnifications. The surface roughness of the membranes was measured by atomic force microscopy (AFM) (XE-100; Park Systems, Korea). The analyzed area was $45 \mu \mathrm{m} \times$ $45 \mu \mathrm{m}$, and the roughness of the membrane surface was shown as mean roughness, $R_{a}$.

The overall porosity $(\varepsilon, \%)$ of the membranes was calculated through gravimetry with DI water as the wetting solvent using the following equation:

$$
\varepsilon=\frac{w_{1}-w_{2}}{d \times A \times \rho} \times 100 \%
$$

where $w_{1}$ is the weight of wet membrane $(\mathrm{kg}), w_{2}$ is the weight of dried membrane $(\mathrm{kg}), d$ is the thickness of the membranes $(\mathrm{m})$, $A$ is the area of the sample $\left(\mathrm{m}^{2}\right)$, and $\rho$ is the density of DI water at $40^{\circ} \mathrm{C}\left(992.2 \mathrm{~kg} / \mathrm{m}^{3}\right)$.

The mean pore radius $\left(r_{m}, \mathrm{~nm}\right)$ was determined using the Guerout-Elford-Ferry equation based on pure water flux and porosity based on the following equation:

$$
r_{m}=\sqrt{\frac{(2.9-1.75 \varepsilon) \times 8 \eta d Q}{A_{m} \times \Delta P \times \varepsilon}}
$$

where $\eta$ refers to the water viscosity (0.0010016 Pa-s), $A_{m}$ is the effective membrane area $\left(\mathrm{m}^{2}\right), Q$ is the volume water flux $\left(\mathrm{m}^{3} / \mathrm{s}\right)$, and $\triangle \mathrm{P}$ is the operating pressure (4 bar).

For evaluation of the surface hydrophilicity of the membranes, a contact angle goniometer (Phoenix 300; Surface Electro Optics Co., USA) was employed using sessile drop methods. For the static sessile drop method, $3 \mu \mathrm{L}$ of DI water was dropped onto the surface of dried membranes using a micro-syringe, and the contact angle was measured.

The mechanical properties of the membranes were measured by using a universal testing machine (UTM) (TO-100-IC; Testone Co., Ltd., Korea). The tensile test was carried out by following the ASTM-D412 method. The sample was cut to dumbbell-shaped tensile specimens of $6 \mathrm{~mm}$ width using a die cutter. The sample was then extended at a constant speed of $20 \mathrm{~mm} / \mathrm{min}$ with a load of $3 \mathrm{kgf}$.

\subsection{Membrane Permeability and Selectivity}

A lab-scale ultrafiltration (UF) experiment (Fig. S1) was carried out to measure the pure water flux and rejection of the membranes. An acryl cell (Millipore Inc., USA) with $18.56 \mathrm{~m}^{2}$ effective membrane area was used for cross-flow filtration. The temperature was kept at $25^{\circ} \mathrm{C}$, and the flow rate was maintained at $400 \mathrm{~cm}$. In order to reach the stable water flux, all the membranes were stabilized at 4 bars for $30 \mathrm{~min}$. DI water was used as feed to measure the pure water flux and a 100 ppm humic acid (HA) (Sigma-Aldrich, USA) solution was used as feed for the rejection test.

The water flux was described via the following equation:

$$
J_{w}=\frac{V}{A_{m} \Delta t}
$$

where $J_{w}$ is the water flux $\left(\mathrm{L} / \mathrm{m}^{2}-\mathrm{h}, \mathrm{LMH}\right), \mathrm{V}$ is the volume of permeated water (L), and $\triangle t$ is the permeation time (h). The rejection of the membranes was determined using the following equation:

$$
\mathrm{R}(\%)=\left(1-\frac{c_{p}}{c_{f}}\right) \times 10
$$

where R (\%) represents the HA rejection, and $C_{p}$ and $C_{f}$ are the concentration of HA in the permeate and feed, respectively. The concentration was measured using a UV/vis spectrophotometer (Optizen POP; Mecasys, Korea) at the wavelength of $290 \mathrm{~nm}$.

\subsection{Fouling Experiment}

To evaluate the antifouling properties of the membranes, three consecutive membranes fouling experiments were conducted at 4 bar using 100 ppm HA solution as feed and the water flux was recorded for $15 \mathrm{~min}$. The fouled membrane was washed with DI water by flushing onto the membrane. The water flux of the membranes was then measured again. Note that the membranes were initially stabilized for $30 \mathrm{~min}$ with DI water at 4 bars before the fouling test. The flux recovery ratio (FRR), total flux loss $\left(R_{t}\right)$, reversible fouling ratio $\left(R_{r}\right)$, and irreversible fouling ratio $\left(R_{i r}\right)$ were calculated using the following equations:

$$
\begin{gathered}
\operatorname{FRR}(\%)=\frac{J_{w, c}}{J_{w, i}} \times 100 \% \\
R_{t}(\%)=\frac{J_{w, i}-J_{w, f}}{J_{w, i}} \times 100 \% \\
R_{r}(\%)=\frac{J_{w, c^{-}} J_{w, f}}{J_{w, i}} \times 100 \% \\
R_{i r}(\%)=\frac{J_{w, i}-J_{w, c}}{J_{w, i}} \times 100 \%
\end{gathered}
$$

where $J_{w, i}, J_{w, f}$, and $J_{w, C}$ represent the water flux of the initial, fouled, and cleaned membrane after HA filtration, respectively, as shown in Fig. S2. Normalized water flux was used to evaluate the anti-fouling properties of the membranes. The measured water flux was divided by the initial water flux to calculate the normalized water flux. 


\section{Results and Discussion}

\subsection{Characterization of HNTs \& f-HNTs}

\subsubsection{Morphology}

The morphologies of the HNTs and f-HNTs were characterized by FE-SEM, as shown in Fig. S3. The HNTs show straight tubular structure open ends with multilayer aluminosilicate sheets which are a typical shape of halloysite nanotube. The HNTs possessed a length of up to $1 \mu \mathrm{m}$ and an outer diameter of 50-100 nm. There might be some transformation of the shape during the functionalization process. However, the f-HNTs were neither folded nor bent after functionalization with APTES. The structure of HNTs and f-HNTs could create some space which acts as channels that can transport [18].

\subsubsection{FTIR}

As can be seen in Fig. 2(a), the peaks at 3,960 $\mathrm{cm}^{-1}$ and 3,622 $\mathrm{cm}^{-1}$ were observed and referred to the stretching absorption of $\mathrm{Al}_{2} \mathrm{OH}$, and the peak at $1,033 \mathrm{~cm}^{-1}$ corresponded to Si-O-Si groups $[19,20]$. The above peaks were shown at the same band position for both the HNTs and f-HNTs, indicating that the basic structure of f-HNTs was not changed after functionalization with APTES. However, in the f-HNT spectrum, a new peak at $1537 \mathrm{~cm}^{-1}$ and at a range of 1,470-1,350 $\mathrm{cm}^{-1}$ were detected for the $\mathrm{N}-\mathrm{H}$ functional group and $\mathrm{C}-\mathrm{H}$ bending in APTES and this reveals that the f-HNTs were functionalized successfully [21].

\subsubsection{XPS}

To further affirm successful functionalization, the XPS spectra of the HNTs and f-HNTs were obtained and are shown in Fig. 2(b). The peaks were clear at $540 \mathrm{eV}, 103 \mathrm{eV}$, and $74 \mathrm{eV}$, which represent $\mathrm{O} 1$ s, Si $2 p$, and $\mathrm{Al} \mathrm{2p}$, respectively. These peaks were attributed to aluminol (Al-OH) and silanol (Si-OH) on both the HNT and f-HNT surfaces. However, a peak of N1s (407 eV) was detected in the spectra of f-HNTs, and the peaks of Si, Al, and

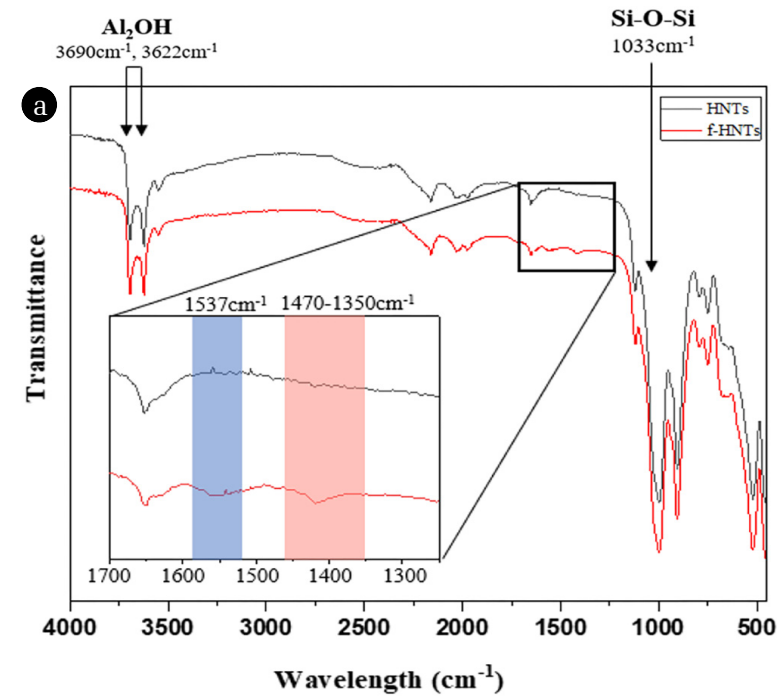

Fig. 2. (a) FTIR and (b) XPS spectra of HNTs and f-HNTs.
$\mathrm{O}$ of $\mathrm{f}-\mathrm{HNT}$ s was more obvious than those in HNTs, indicating that the covalent bonds were formed between HNTs and APTES [18]. The peaks of the XPS spectra of $\mathrm{Al} 2 \mathrm{p}, \mathrm{Si} 2 \mathrm{p}$, and $\mathrm{O} 1 \mathrm{~s}$ are drawn in Fig. S4. As shown in Fig. S4, the binding energy peak value of $\mathrm{Al}$ atom is moved from $74.98 \mathrm{eV}$ to $74.78 \mathrm{eV}$, and that of $\mathrm{Si}$ atom is also changed from $103.38 \mathrm{eV}$ to $103.08 \mathrm{eV}$ after functionalization of HNTs with APTES. Also, the binding energy of $\mathrm{O}$ atom at peak value moves from $532.38 \mathrm{eV}$ to $532.18 \mathrm{eV}$. These moved binding energy values for $\mathrm{Al}, \mathrm{Si}$ and $\mathrm{O}$ atoms may be related to the covalent bonding by coupling reaction between APTES and HNTs [22].

\subsection{Membrane Characterization}

\subsubsection{Morphology}

Surface and cross-sectional images of the membranes were taken by FE-SEM. The surface of the fabricated membranes is shown in Fig. 3. The HNTs and f-HNTs were observed on the surface of the membranes, as indicated by the circle. As can be seen, the number of HNTs and f-HNTs on the surface increased with the addition of HNTs and f-HNTs. Even though a number of HNTs and f-HNTs were embedded in the membrane, which might cause some defects such as a pinhole or a small tear due to agglomeration of an excessive amount of HNTs and f-HNTs, there were no observable defects on the surface. As shown in Fig. S5, all the membranes possessed an asymmetric porous structure with a thin dense upper skin layer and a finger-like porous sub-layer, which are the typical morphologies of a membrane fabricated via immersion precipitation phase inversion [23]. The overall thickness of the fabricated membranes was controlled in a range of 90-110 $\mu \mathrm{m}$.

Fig. S6 shows the roughness of the surface of the fabricated membranes as average roughness $\left(R_{a}\right)$. With the addition of HNTs and f-HNTs into the membranes, the average roughness decreased from $16.21 \mathrm{~nm}$ (PES) to $12.14 \mathrm{~nm}$ (HNT2.0) and $11.55 \mathrm{~nm}$ (f-HNT2.0). The introduction of HNTs and f-HNTs contributes to smoothing of the surface structure of the membranes due to the spontaneous migration of HNTs and f-HNTs to the membrane/water

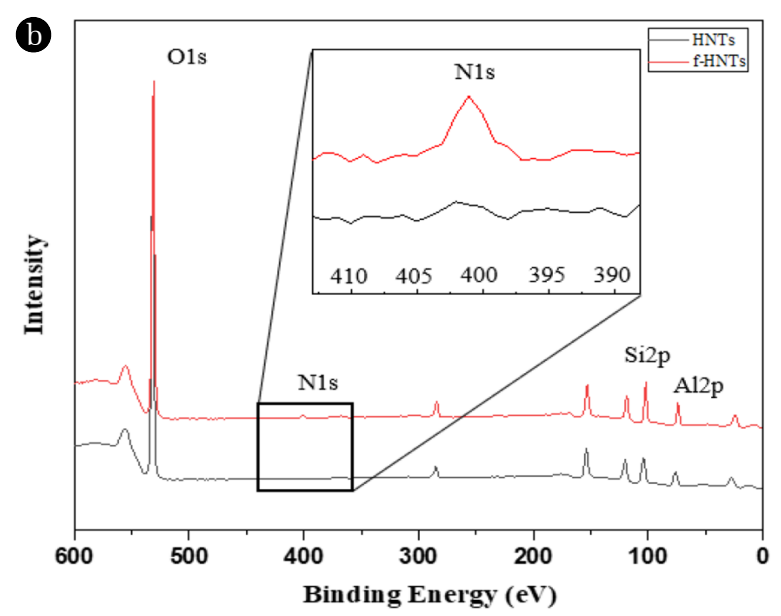



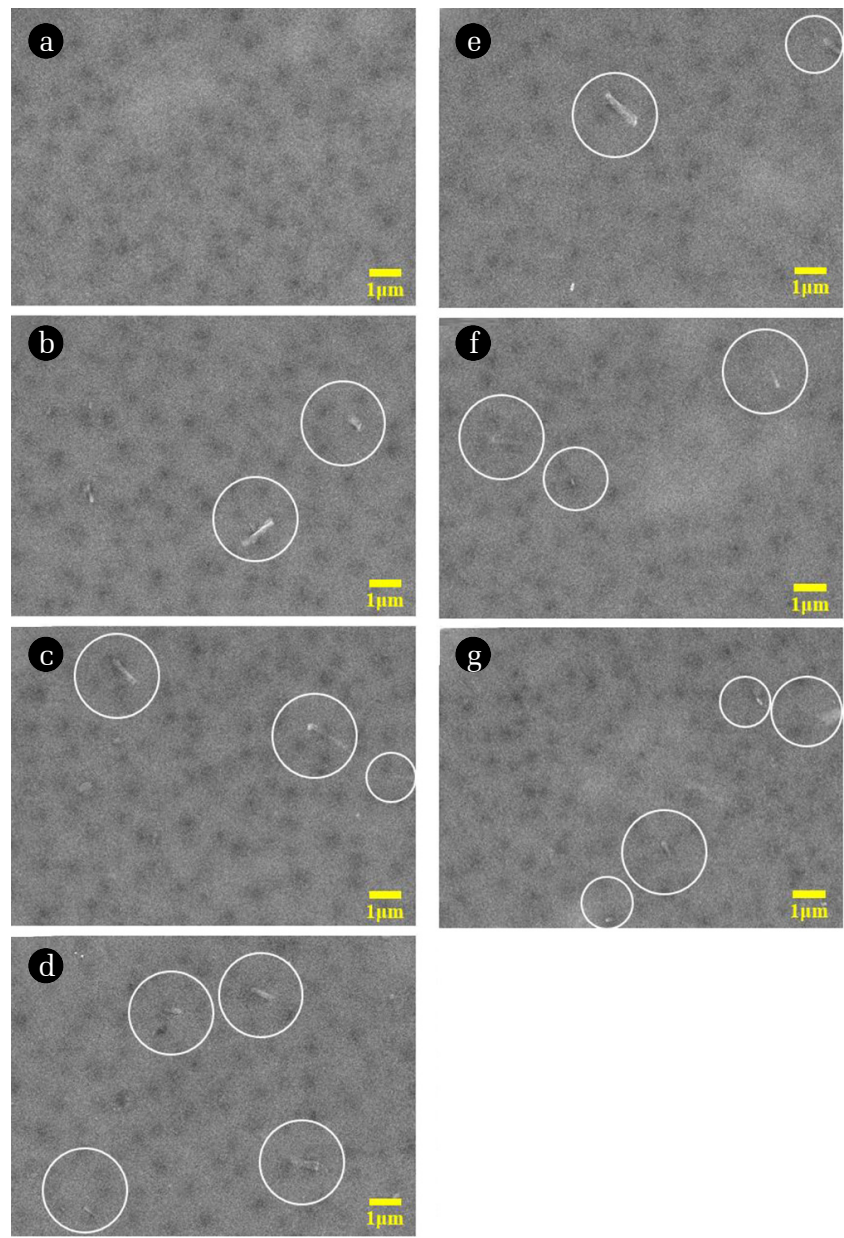

Fig. 3. FE-SEM images of fabricated membrane surface of (a) PES, (b) HNT0.5, (c) HNT1.0, (d) HNT2.0, (e) f-HNT0.5, (f) f-HNT1.0, (g) f-HNT2.0 captured at 10,000 x magnification.

interface during the phase inversion process, and this migration helps to fill the valleys that are present on the membrane surface [18]. It is well established that impurities were likely to be absorbed in the valleys of membrane, leading to clogging of the valleys [24]. In addition, APTES also played a significant role in strengthening the interfacial interaction between the membrane and f-HNTs. Many studies have proven that membranes with a lower roughness exhibit a better antifouling property because the foulant in wastewater may not easily accumulate on the surface of the membranes [25, 26].

\subsubsection{Intrinsic properties}

The intrinsic properties (i.e., pore properties, hydrophilicity, and mechanical properties) of the synthesized membranes were evaluated and the results are shown in Fig. 4 and Table 2.

The effects of the HNT and f-HNT content on the porosity and mean pore size of the membranes are listed in Table 2. All the membranes showed similar porosity percentage of around $79 \%$. The mean pore size of the membrane was increased with the addition of HNTs and f-HNTs due to the hydrophilicity of the membranes because the solution with higher hydrophilicity will
Table 2. The Overall Porosity and Mean Pore Size of the Fabricated Membranes

\begin{tabular}{lcc}
\hline & Overall porosity $(\%)$ & Mean pore size $(\mathbf{n m})$ \\
\hline PES & $79.09 \pm 0.16$ & 6.67 \\
HNT0.5 & $79.47 \pm 0.37$ & 10.85 \\
HNT1.0 & $79.75 \pm 0.63$ & 13.25 \\
HNT2.0 & $79.76 \pm 0.67$ & 15.39 \\
f-HNT0.5 & $79.39 \pm 0.43$ & 10.26 \\
f-HNT1.0 & $79.32 \pm 1.01$ & 11.77 \\
f-HNT2.0 & $79.38 \pm 0.60$ & 14.86 \\
\hline
\end{tabular}
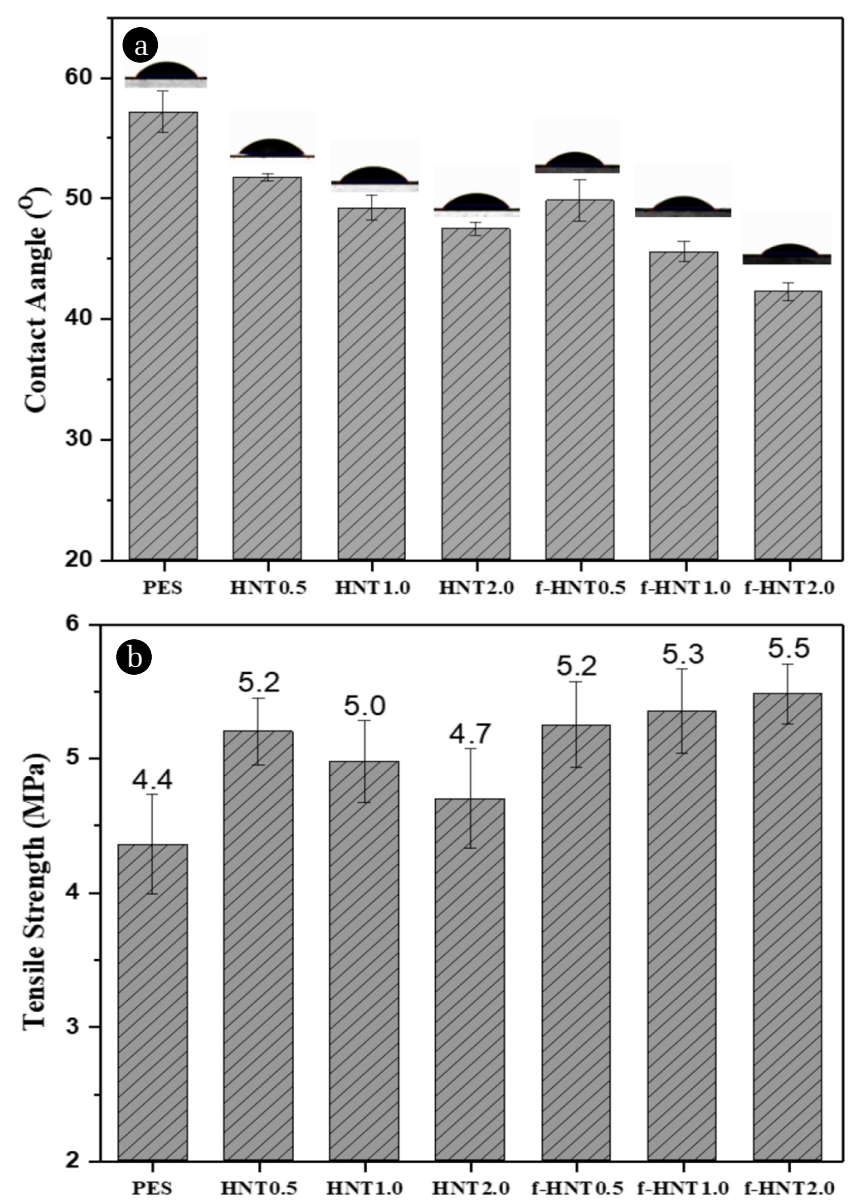

Fig. 4. (a) Contact angle and (b) tensile strength of fabricated membranes.

lead to a faster exchange rate between the solvent and non-solvent (DI water), resulting in large pore size [27]. The PES membrane showed a mean pore size of $6.67 \mathrm{~nm}$, and the mean pore sizes of the membranes with HNTs were $10.85 \mathrm{~nm}$ (HNT0.5), 13.25 $\mathrm{nm}$ (HNT1.0), and $15.39 \mathrm{~nm}$ (HNT2.0). The membranes with f-HNTs showed the same trend as those with HNTs; however, the mean pore sizes were slightly smaller than those of the HNTs: 10.26 nm (f-HNT0.5), $11.77 \mathrm{~nm}$ (f-HNT1.0) and $14.86 \mathrm{~nm}$ (f-HNT2.0). This indicates that the interfacial gap between the f-HNT and the polymer matrix decreased because the functionalization of 
HNTs provided more bonding between the polymer and the f-HNTs, leading to improved interfacial interaction. Thus, the mean pore size of the membranes with f-HNTs was smaller than that of the membranes with HNTs [28].

The contact angle between the surface of the fabricated membranes and DI water was measured and the results are shown in Fig. 4(a). The contact angle is an effective indicator of hydrophilicity of the membrane surface. A higher hydrophilic surface shows a smaller contact angle. The PES membrane exhibited the highest contact angle, $57.1^{\circ}$, corresponding to the lowest hydrophilicity. The contact angle of the composite membranes with HNTs gradually decreased to $51.7^{\circ}$ (HNT0.5), 49.2 ${ }^{\circ}$ (HNT1.0), and $47.4^{\circ}$ (HNT2.0), since the HNTs are intrinsically hydrophilic owing to the functional groups of $-\mathrm{OH}$ on their surface. The affinity of the f-HNT loaded polymeric matrix to water molecules was further improved because the f-HNTs have amine functional groups $\left(-\mathrm{NH}_{2}\right)$ on the surface, which can provide more hydrogen bonding.

Fig. 4(b) shows the influence of the HNTs and f-HNTs on the mechanical properties of the membranes measured by UTM. The results showed that the addition of HNTs and f-HNTs increased the tensile strength because HNTs and f-HNTs played the role of nanofiller. When HNTs were blended at $0.5 \%$, the tensile strength was increased from 4.3 MPa (PES) to 5.2 MPa; however, with an increase of the HNT content, the tensile strength decreased to 5.0 MPa (HNT1.0) and 4.7 MPa (HNT2.0). This phenomenon is attributed to the agglomeration of HNTs in the membranes, which adversely affected the improvement in the mechanical properties caused by the presence of HNTs [29, 30]. On the other hand, the membranes with f-HNTs showed a tendency of increased tensile strength, with values of $5.2 \mathrm{MPa}$ (f-HNT0.5), $5.3 \mathrm{MPa}$ (f-HNT1.0), and 5.5 MPa (f-HNT2.0), even though agglomeration of f-HNTs may have occurred. This is because the interfacial adhesion between the f-HNTs and the polymer matrix were improved due to increased hydrogen bonding by the amine group $\left(-\mathrm{NH}_{2}\right)$ on the surface of the f-HNTs [28].

\subsection{Membrane Permeability and Selectivity}

Fig. 5 shows the pure water flux and HA rejection of the membranes. The pure water flux of the fabricated membranes was dramatically improved with increased amounts of HNTs and f-HNTs without a trade-off relation between water flux and rejection. In general, water flux and rejection have a trade-off relation, because increased pore size facilitates water transport, and simultaneously contaminants also can pass through the pores. In this research, although the contents of HNTs and f-HNTs in the membranes were different and the mean pore size increased with the addition of HNTs and f-HNTs, all the membranes showed a high HA rejection percentage of over 98\%. The rejection of the PES membrane was $98.3 \%$, and the membranes with HNTs showed rejection of 98.7\% (HNT0.5), 99.2\% (HNT1.0), and 99.3\% (HNT2.0). The membranes with f-HNTs showed either similar or relatively higher rejection than the membranes with HNTs at 99.3\% (f-HNT0.5), 99.2\% (f-HNT1.0), and $99.3 \%$ (f-HNT2.0), and obviously higher than that of PES membrane. This is due to the improved hydrophilicity that consequently enhanced the ability of the membrane surface to repel HA [31].

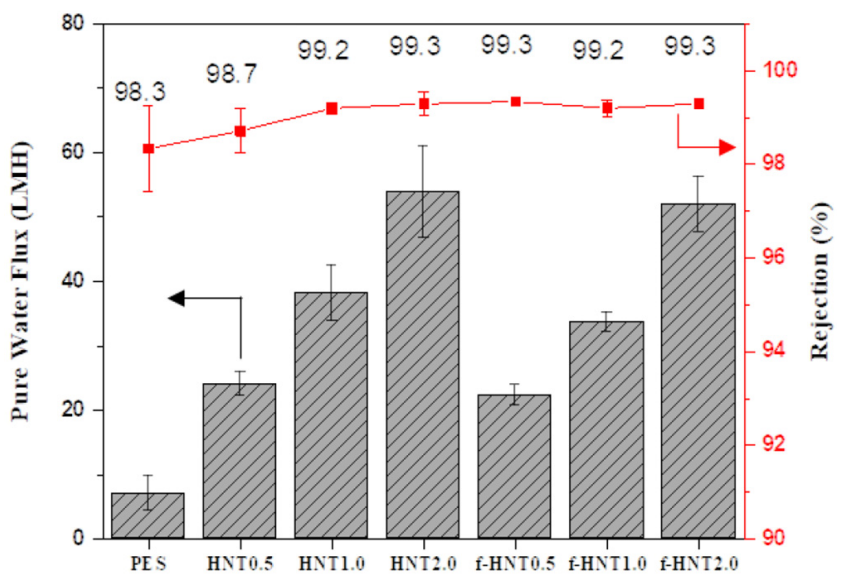

Fig. 5. Pure water flux and $\mathrm{HA}$ rejection of fabricated membranes.

The fabricated membranes with HNTs showed higher pure water flux of 24.0 LMH (HNT0.5), 38.2 LMH (HNT1.0), and 53.8 LMH (HNT2.0) than the pure water flux of 7.1 LMH (PES). This is because the improved mean pore size helps the water permeate through the membranes. In addition, the formation of interfacial gaps between the HNT and the polymer matrix also enhances the water flux because they act as water channels, and increased hydrophilicity also affects the pure water flux owing to the presence of a large amount of hydrophilic functional groups on the surface of the HNTs and f-HNTs [27]. Also, the porous structure of the HNTs helps the water permeate through capillary action [32]. However, the membranes with HNTs and f-HNTs showed similar pure water flux even though the hydrophilicity of the membranes with f-HNTs was higher than that of the membranes with HNTs. As mentioned above, this is because the interfacial gap was decreased due to the functionalization of HNTs, which leads to decreased mean pore size of the membranes.

\subsection{Anti-fouling Properties}

To compare the effect of the addition of HNTs and f-HNTs on the fouling resistance of the membranes, antifouling experiments were carried out using PES, HNT2.0, and f-HNT2.0 membranes. The flux decline was due to the accumulation of HA on the membrane surface at the initial stage of filtration, as shown in Fig. 6(a). After HA solution filtration for $15 \mathrm{~min}$, the membrane was cleaned using DI water, and the water flux was recovered. The decline in the flux of PES was significant compared to HNT2.0 and f-HNT2.0. It is also expressed as $R_{t}$ in the order f-HNT2.0 $<$ HNT2.0 $<$ PES $(13.4 \%<17.6 \%<29.1 \%$, respectively), as can be seen in Fig. 6(b). This is due to the relatively rough and hydrophobic surface of the membranes. The membrane with f-HNTs showed the highest FRR of $95.0 \%$ whereas the FRR was $88.6 \%$ and $77.7 \%$ for HNT2.0 and PES, respectively. In this case, the enhanced hydrophilicity plays a key role during HA filtration. The hydrophobic interaction between the HA and membrane surface allows the hydrophilic membrane to maintain its water flux during filtration, because the hydrophilic membrane repels HA from the membrane surface [33]. In addition, as the hydrophilicity is increased, a hydrated layer can be easily formed on the surface and this hydrated 

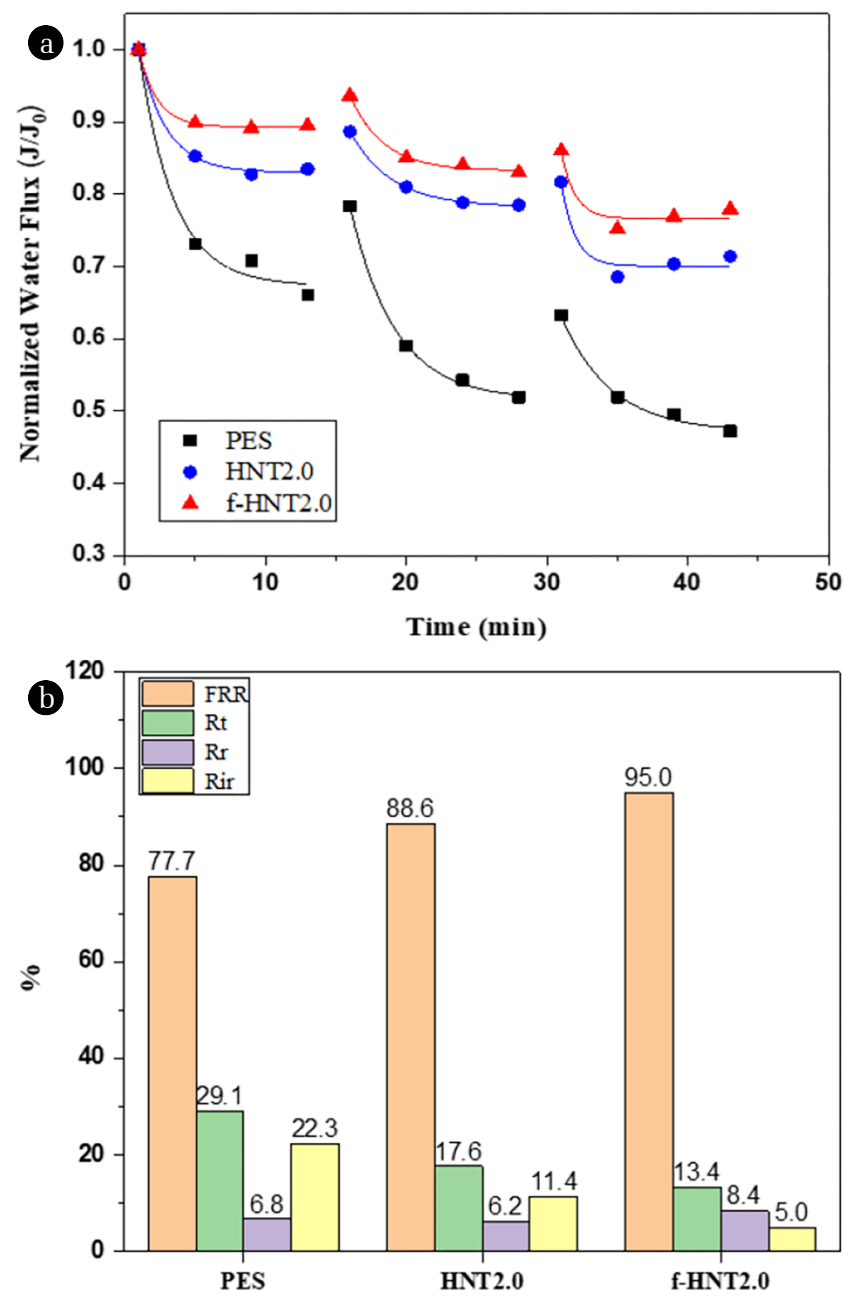

Fig. 6. (a) HA fouling experiment result and (b) Antifouling properties of the membranes (PES, HNT2.0, f-HNT2.0).

layer can prevent the adsorption and deposition of HA onto the membrane surface and thus reduce membrane fouling [31]. As shown in Fig. 6(b), $R_{i r}$ was also observed similarly, in the order f-HNT2.0 < HNT2.0 < PES $(5.0 \%<11.4 \%<22.3 \%$, respectively). This is due to the fact that foulants in the feed are easily trapped in the valleys present on the rough membrane surface and hence such membranes were difficult to clean and result in irreversible fouling.

The above results demonstrate that blending HNTs and f-HNTs in polymeric membranes can increase the fouling resistance to HA. In addition, functionalization of HNTs with APTES also can improve the antifouling properties of the membrane. In order to support this result, various fouling experiments should be conducted using inorganic foulants or bio-foulants under different conditions.

\section{Conclusion}

In this study, HNTs were functionalized with APTES in order to improve the hydrophilicity and the interfacial interaction be- tween the HNTs and a polymer matrix. Nanocomposite membranes with HNTs and f-HNTs were fabricated via the phase inversion method, and the characteristics and performance of the membranes were evaluated and compared.

The conclusions are as follows:

(1) HNTs were successfully functionalized with APTES and this was confirmed by XPS and FTIR results.

(2) The contact angle of membranes with HNTs and f-HNT decreased, which is attributed to higher hydrophilicity relative to PES. Also, the hydrophilicity of the membranes with f-HNTs was further improved due to its hydrophilic functional groups.

(3) The mechanical properties of the membranes with HNTs and f-HNTs were improved. With an increase of f-HNT content of the membranes, the tensile strength was enhanced whereas the membranes with HNTs showed decreasing tensile strength. This is because the interfacial adhesion between the f-HNTs and the polymer matrix was improved.

(4) The pure water flux of the fabricated membranes was dramatically improved with an increase of HNT and f-HNT content without a trade-off relation between water flux and rejection. This is due to the improved hydrophilicity and increased mean pore size of the membranes.

(5) The membranes with f-HNTs showed better antifouling properties than the membranes with HNTs because of increased hydrophilicity, which leads to the formation of thicker hydrated layer that prevents the adsorption and deposition of HA. Moreover, the low roughness of the membrane surface also played a role in the prevention of HA accumulation on the membrane surface.

\section{Acknowledgment}

This work was supported by the project of "Development of key membranes for high efficiency seawater desalination" supported by the Korea Research Institute of Chemical Technology (KK1802-B00).

\section{Author Contributions}

S.P. (MS student) has performed all the experiments and written the manuscript. E.Y. (Ph.D. student) has provided revisions to scientific content of manuscript. H.P. (Ph.D.) and H.C. (Professor) have given direction on this work and revised the manuscript for several times with critical comments.

\section{References}

1. Geise GM, Lee HS, Miller DJ, Freeman BD, McGrath JE, Paul DR. Water purification by membranes: The role of polymer science. J. Polym. Sci. 2010;48:1685-1718.

2. Lalia BS, Kochkodan V, Hashaikeh R, Hilal N. A review on membrane fabrication: Structure, properties and performance relationship. Desalination 2013;326:77-95.

3. Lee A, Elam JW, Darling SB. Membrane materials for water 
purification: design, development, and application. Environ. Sci. (Camb) 2016;2:17-42.

4. Pendergast MM, Hoek EMV. A review of water treatment membrane nanotechnologies. Energ. Environ. Sci. 2011;4:1946.

5. Giwa A, Akther N, Dufour V, Hasan SW. A critical review on recent polymeric and nano-enhanced membranes for reverse osmosis. RSC Adv. 2016;6:8134-8163.

6. Celik E, Park H, Choi H, Choi H. Carbon nanotube blended polyethersulfone membranes for fouling control in water treatment. Water Res. 2011;45:274-282.

7. Liang S, Xiao K, Mo Y, Huang X. A novel ZnO nanoparticle blended polyvinylidene fluoride membrane for anti-irreversible fouling. J. Memb. Sci. 2012;394-395:184-192.

8. Zhang J, Xu Z, Shan M, et al. Synergetic effects of oxidized carbon nanotubes and graphene oxide on fouling control and anti-fouling mechanism of polyvinylidene fluoride ultrafiltration membranes. J. Memb. Sci. 2013;448:81-92.

9. Maximous N, Nakhla G, Wan W, Wong K. Preparation, characterization and performance of $\mathrm{Al} 2 \mathrm{O} 3 / \mathrm{PES}$ membrane for wastewater filtration. J. Memb. Sci. 2009;341:67-75.

10. Luo M-l, Tang W, Zhao J-q, Pu C-s. Hydrophilic modification of poly(ether sulfone) used $\mathrm{TiO}_{2}$ nanoparticles by a sol-gel process. J. Mater. Process. Technol. 2006;172:431-436.

11. Taurozzi JS, Arul H, Bosak VZ, et al. Effect of filler incorporation route on the properties of polysulfone-silver nanocomposite membranes of different porosities. J. Memb. Sci. 2008;325: 58-68.

12. Asempour F, Akbari S, Bai D, Emadzadeh D, Matsuura T, Kruczek B, Improvement of stability and performance of functionalized halloysite nano tubes-based thin film nanocomposite membranes. J. Memb. Sci. 2018;563:470-480.

13. Kuroda Y, Ito K, Itabashi K, Kuroda K. One-step exfoliation of kaolinites and their transformation into nanoscrolls. Langmuir 2011;27:2028-2035.

14. Yuan P, Southon PD, Liu Z, et al. Functionalization of halloysite clay nanotubes by grafting with $\gamma$-Aminopropyltriethoxysilane. J. Phys. Chem. C 2008;112:15742-15751.

15. Kamble R, Ghag M, Gaikawad S, Panda BK. Halloysite nanotubes and applications: A review. J. Adv. Sci. Res. 2012;3:25-29.

16. Yan S, Yang Y, Song L, Qi X, Xue Y, Fan B. Influence of 3-aminopropyltriethoxysilane- graphite oxide composite on thermal stability and mechanical property of polyethersulfone. High Perform. Polym. 2016;29:960-975.

17. Xie Y, Hill CAS, Xiao Z, Militz H, Mai C. Silane coupling agents used for natural fiber/polymer composites: A review. Compos. Part A. 2010;48:806-819.

18. Zeng G, He Y, Zhan Y, et al. Novel polyvinylidene fluoride nanofiltration membrane blended with functionalized halloysite nanotubes for dye and heavy metal ions removal. J. Hazard. Mater. 2016;317:60-72.

19. Yu H, Zhang Y, Sun X, Liu J, Zhang H. Improving the antifouling property of polyethersulfone ultrafiltration membrane by in- corporation of dextran grafted halloysite nanotubes. Chem. Eng. J. 2014;237:322-328.

20. He Y, Xu W, Tang R, Zhang C, Yang Q. pH-Responsive nanovalves based on encapsulated halloysite for the controlled release of a corrosion inhibitor in epoxy coating. RSC $A d v$. 2015;5:90609-90620.

21. Khan J, Siddiq M, Akram B, Ashraf MA. In-situ synthesis of $\mathrm{CuO}$ nanoparticles in P(NIPAM-co-AAA) microgel, structural characterization, catalytic and biological applications. Arab. J. Chem.2018;11:897-909.

22. Zhang Y, He X, Ouyang J, Yang H. Palladium nanoparticles deposited on silanized halloysite nanotubes: Synthesis, characterization and enhanced catalytic property. Sci. Rep. 2013;3: 2948.

23. Loeb S, Sourirajan S. Sea water demineralization by means of an osmotic membrane. Adv. Chem. Ser. 1962;38:117-132.

24. Chen Y, Zhang Y, Liu J, Zhang H, Wang K. Preparation and antibacterial property of polyethersulfone ultrafiltration hybrid membrane containing halloysite nanotubes loaded with copper ions. Chem. Eng. J. 2012;210:298-308.

25. Zhao Q, Hou J, Shen J, Liu J, Zhang Y. Long-lasting antibacterial behavior of a novel mixed matrix water purification membrane. J. Mater. Chem. A 2015;3:18696-18705.

26. Yu L, Zhang Y, Zhang H, Liu J. Development of a molecular separation membrane for efficient separation of low-molecular-weight organics and salts. Desalination 2015;359:176-185.

27. Mishra G, Mukhopadhyay M. Enhanced antifouling performance of halloysite nanotubes (HNTs) blended poly(vinyl chloride) (PVC/HNTs) ultrafiltration membranes: For water treatment. J. Ind. Eng. Chem. 2018;63:366-379.

28. Munirathnamma LM, Ravikumar HB. Microstructural characterization of short glass fibre reinforced polyethersulfone composites: A positron lifetime study. J. Appl. Polym. Sci. 2016;133.

29. Gaaz TS, Sulong AB, Ansari MNM, Kadhum AAH, Al-Amiery AA, Al-Furjan MSH. Effect of halloysite nanotubes loading on thermo-mechanical and morphological properties of polyurethane nanocomposites. Mater. Technol. 2016;32:430-442.

30. Cai N, Dai Q, Wang Z, Luo X, Xue Y, Yu F. Toughening of electrospun poly(L-lactic acid) nanofiber scaffolds with unidirectionally aligned halloysite nanotubes. J. Mater. Sci. 2014;50:1435-1445.

31. Kumar R, Ismail AF. Fouling control on microfiltration/ultrafiltration membranes: Effects of morphology, hydrophilicity, and charge. J. Appl. Polym. Sci. 2015;132.

32. Yuan P, Tan D, Annabi-Bergaya F. Properties and applications of halloysite nanotubes: Recent research advances and future prospects. Appl. Clay Sci. 2015;112-113:75-93.

33. Son M, Kim H, Jung J, Jo S, Choi H. Influence of extreme concentrations of hydrophilic pore-former on reinforced polyethersulfone ultrafiltration membranes for reduction of humic acid fouling. Chemosphere 2017;179:194-201. 